\title{
MINING PUBLIC OPINION ON RIDE-HAILING SERVICE PROVIDERS USING ASPECT-BASED SENTIMENT ANALYSIS
}

\author{
Isti Surjandari ${ }^{1 *}$, Reggia Aldiana Wayasti ${ }^{1}$, Zulkarnain $^{1}$, Enrico Laoh $^{1}$, \\ Annisa Marlin Masbar Rus ${ }^{1}$, Irfan Prawiradinata ${ }^{2}$ \\ ${ }^{1}$ Department of Industrial Engineering, Faculty of Engineering, Universitas Indonesia, Kampus UI \\ Depok, Depok 16424, Indonesia \\ ${ }^{2}$ The Boston Consulting Group (BCG), Sampoerna Strategic Square, Jakarta 12930, Indonesia
}

(Received: February 2019 / Revised: May 2019 / Accepted: June 2019)

\begin{abstract}
The use of ride-hailing services as a solution to current transportation problems is currently attracting much attention. Their benefits and convenience mean many people use them in their everyday lives and discuss them in the social media. As a result, ride-hailing service providers utilize social media to capture customers' opinions and to market their services. If these opinions and comments are analyzed, service providers can obtain feedback to evaluate their services in order to achieve customer satisfaction. This study combines the text mining approach, in the form of aspect-based sentiment analysis to identify topics in customer opinions and their sentiments, with scoring of ride-hailing service providers in general, and more specifically based on the topics and sentiments. The study analyzes customers' opinions on Twitter of three ride-hailing service providers. Text data were classified based on six topics derived from the topic modeling process, along with the sentiments expressed on them. Scoring of the three ride-hailing service providers was based on the number of positive and negative comments in relation to each topic, as well as overall comments. The results of the study can be used as input to evaluate and improve the service in Indonesia, thus the customer satisfaction and loyalty can be maintained and improved.
\end{abstract}

Keywords: Aspect-based sentiment analysis; Latent Dirichlet Allocation; Net Reputation Score; Ride-hailing service; Support Vector Machine; Text mining

\section{INTRODUCTION}

Currently, social media is continuously developing, along with technological advancements. It is now used for various purposes as well as communicating and socializing, such as seeking entertainment and information (He et al., 2013). This is because social media provides an easy way to create and exchange user-generated content (UGC). Social media users can actively participate to create and share content in the form of text messages, photos, videos, amongst others. This content can be accessed and responded to instantly by other users. The volume of shared content increases over time at a fast rate, resulting in high dimensional data (Tang \& Liu, 2014).

The amount of information available enables social media to play an important role in the electronic word of mouth (e-WOM) process. Content that includes opinions on or reviews of products or services has an important influence in shaping public perceptions, building product or service reputation, and helping customers make purchasing decisions, which all leads to 
increased sales and profitability (Philander \& Zhong, 2016). Quick distribution of the content allows a product or service to be recognized, so that the responses and comments from the public can be accessed and monitored over time.

Among the existing social media platforms, Twitter is one of the most popular microblogs. Twitter users can share content in a tweet and interact with fellow users. Every day, more than half a billion tweets are posted, which means that much content is shared. The fast way of sharing and exchanging UGC on Twitter makes the e-WOM process effective (Philander \& Zhong, 2016). Customers can obtain information about products or services quickly, while companies can monitor and analyze their comments to ascertain the advantages and disadvantages of their products or services.

Comments from customers in social media such as Twitter can generate insight for companies to develop further strategies for their products or services. To handle and extract information from a large number of posts and various writing styles, the text mining approach can be applied. One text mining application is sentiment analysis which processes opinionated posts and groups them based on their sentiment (Surjandari et al., 2015). There is also the aspect-based sentiment analysis technique, which identifies related topics to the object being reviewed before grouping the sentiments for each topic (Marrese-Taylor et al., 2014). This technique produces a more detailed grouping scheme and is more helpful to users, because the features that receive positive and negative sentiments can be defined.

This study aims to analyze customers' opinions on Twitter by defining the topics discussed and their respective sentiments. Ride-hailing service providers in Indonesia were chosen to be the object of the study, since more people use these services daily. Many users share their experience, compliments and complaints about the services on Twitter. Therefore, these kinds of tweets can be utilized by the service providers to develop improvement strategies so that they can continue to provide the best service and increase customer loyalty. The study also applies a scoring scheme for the ride-hailing service providers based on the sentiment analysis results to help them decide improvement priorities.

\section{RELATED WORK}

Research employing aspect-based sentiment analysis has been conducted to extract public opinion from various sources. Marrese-Taylor et al. (2014) applied aspect-based sentiment analysis to analyze hotel and restaurant reviews. In addition, Colace et al. used it to analyze movie reviews, social media posts and online learning websites (Colace et al., 2015).

The first step in aspect-based analysis is to extract the topics. One of the methods to do this is topic modeling, with several studies conducted related to this. Duan et al. applied the Latent Dirichlet Allocation (LDA) method combined with user interest to recommend topics of interest in microblogs (Duan et al., 2015). Tong and Zhang also used LDA to search, explore and recommend articles for Wikipedia users, and to analyze users' interests on Twitter (Tong \& Zhang, 2016). Lim and Buntine utilized hashtags, mentions, emoticons and sentiment words in Twitter for topic modeling purposes, using the LDA-based opinion model method, with improved opinion prediction by directly modeling the interaction between target and opinion (Lim \& Buntine, 2012). Putri and Kusumaningrum identified review tendencies and classified sentiment from TripAdvisor using LDA with different parameters (Putri \& Kusumaningrum, 2017).

There have also been studies related to sentiment analysis. Research by Surjandari et al. analyzed tweets about staple food prices and their changes in Indonesia, classifying them into positive and negative categories (Surjandari et al., 2015). On the other hand, Surjandari et al. (Surjandari et al., 2016) classified complaints from the National Complaint Handling System in Indonesia into six predefined classes and clustered the data in each class to define more specific topics. 
Prameswari et al. applied sentiment analysis for tourism purposes by classifying reviews on TripAdvisor of two of Indonesia's priority tourist destinations into five categories with their polarities, and defining the representative sentences using text summarization (Prameswari et al., 2017).

Some research has also applied certain methods to assess products or services from social media data. Competitive analysis was used by He et al. to compare pizza chains based on the number of posts, likes, retweets, shares and followers (He et al., 2013). Vidya et al. used net brand reputation to compare positive and negative sentiments from the users of mobile phone providers (Vidya et al., 2015). Ratio score calculations were made by Philander and Zhong to assess hotels and resorts based on the number of positive and negative sentiments on Twitter (Philander \& Zhong, 2016). Liu et al. applied the intuitionistic fuzzy approach to rank products from web reviews (Liu et al., 2017).

Previously, research combining sentiment analysis and product or service assessment has been based on predetermined aspects. These aspects can be less representative, which can affect the results. Hence, this study combines aspect-based analysis with the assessment method to produce a score and compare the three providers of ride-hailing services in Indonesia. The assessment itself can be made both as a whole and based on the topics discussed by customers.

\section{RESEARCH METHODOLOGY}

Text mining is a feature of data mining which is applied to extract hidden information from unstructured text data (Surjandari et al., 2015). Due to the increasing number of text data from various sources, research related to data mining is also increasing. One of its applications is in business, because text mining can produce deep and meaningful analysis and information (Chakraborty et al., 2013).

Three steps are involved in obtaining information by applying text mining. The first is to collect the text data from certain sources. To make the data more structured and easier to process, a preprocessing step is employed. Finally, the data are processed by applying algorithms and different methods to obtain results that are expected and in line with the objective (Miner et al., 2012).

Text mining consists of unsupervised and supervised methods. This study uses the unsupervised method for the topic modeling and the supervised method for the sentiment analysis. The classes of sentiment analysis were derived from the results of topic modeling, while the sentiments for each topic which were divided into positive and negative groups.

\subsection{Data Gathering}

Comments, stories and complaints on Twitter related to three ride-hailing service providers in Indonesia from customers were used as the study data in. The tweets were collected by web scraping using Twitter API. The ones used were those posted from the first week of January 2018 to the second week of March 2018. The process of tweets scraping was conducted weekly as a condition of Twitter API is that it can only obtain tweets for a week-long period.

All the tweets collected went through selection and screening processes. These processes were conducted to ensure that the tweets were all in Indonesian, with those removed which were not from customers, were unnecessary, or were irrelevant. All the qualifying tweets then went through a pre-processing step.

\subsection{Data Pre-processing}

There are no rules for posting text on Twitter, so it can be done freely, which leads to a diversity of writing formats. Text data with diverse formats need to go through several pre-processing steps to make them more structured and able to be subsequently processed using the text mining method. The pre-processing steps can be performed manually or by using the appropriate 
software. While the former is more likely to take more time to complete, the availability of the latter for Indonesia is limited in terms of reliability and capability. However, the importance of this phase is crucial for the following process to be relevant. Therefore, careful attention was paid and more time spent on this phase.

Six text pre-processing steps were performed. The first was spelling normalization to change all the words into standard Indonesian language. Next, case folding was conducted to convert all the letters into lowercase. Filtering was also conducted to remove punctuation marks, symbols, hashtags (\#), URLs, emoticons, stop words, and other unrelated words with no particular meaning. All the words were changed to their root form in a stemming step. Tokenization was then employed to split the sentences into terms called tokens.

The next step was to transform text data into numerical form by matrix formation. Three matrices were formed: Term Frequency (TF), Inverse Document Frequency (IDF), and Singular Value Decomposition (SVD). The TF matrix, as shown in Table 1, indicates how many times a word or token appears in a document. Based on the occurrence, the IDF matrix was used to calculate the weight of each word by dividing its frequency with the total number of words in the corpus or a document, which in this case is a Twitter posting, as illustrated in Table 2. Next, the dimension of the TF-IDF matrix and data sparsity were reduced in SVD by converting them into the concepts shown in Table 3. By taking these pre-processing steps, the performance of the algorithm can be improved (Prameswari et al., 2017).

Table 1 Term frequency matrix

\begin{tabular}{cccccc}
\hline & $\begin{array}{c}1 \\
\text { accept }\end{array}$ & $\begin{array}{c}2 \\
\text { account }\end{array}$ & $\begin{array}{c}3 \\
\text { active }\end{array}$ & $\begin{array}{c}4 \\
\text { address }\end{array}$ & $\begin{array}{c}5 \\
\text { application }\end{array}$ \\
\hline 1 & 1 & & & & 1 \\
2 & & 1 & 1 & & 1 \\
3 & & & & 1 & 1 \\
4 & 1 & 1 & & & 1 \\
5 & & & 1 & & \\
6 & & & & & \\
\hline
\end{tabular}

Table 2 Inverse document frequency matrix

\begin{tabular}{cccccc}
\hline & $\begin{array}{c}1 \\
\text { accept }\end{array}$ & $\begin{array}{c}2 \\
\text { account }\end{array}$ & $\begin{array}{c}3 \\
\text { active }\end{array}$ & $\begin{array}{c}4 \\
\text { address }\end{array}$ & $\begin{array}{c}5 \\
\text { application }\end{array}$ \\
\hline 1 & 2.17548 & & & & 2.20827 \\
2 & & 3.07789 & 3.32435 & & \\
3 & & & & 4.16255 & 2.20827 \\
4 & 2.17548 & 3.07789 & & & 2.20827 \\
5 & & & 3.32435 & & 2.20827 \\
6 & & & & & \\
\hline
\end{tabular}

Table 3 Singular value decomposition matrix

\begin{tabular}{ccccc}
\hline Document & Concept 1 & Concept 2 & Concept 3 & Concept 4 \\
\hline 1 & 0.0233 & 0.0165 & -0.0178 & 0.0116 \\
2 & 0.0242 & -0.0275 & -0.0816 & -0.0073 \\
3 & 0.0145 & -0.0154 & 0.0156 & -0.0186 \\
4 & 0.0103 & -0.0067 & 0.0098 & -0.0164 \\
5 & 0.0291 & 0.0271 & -0.0027 & 0.0337 \\
6 & 0.0213 & -0.0242 & 0.0021 & 0.0171 \\
\hline
\end{tabular}




\subsection{Topic Modeling}

Latent Dirichlet Allocation (LDA) was also employed, which is a method for probabilistic topic modeling. Its main task is to find topics which are distributed in the corpus or a document with high probability. LDA uses the Bag of Words (BoW) assumption that does not counts on the order of words (Blei, 2012).

There are several parameters in LDA, as shown in the model in Figure 1 (Blei, 2012). In the model, $D$ is the number of documents, while $N$ is the number of words in the document. The parameters that have to be defined are the number of topics $(K)$, number of iterations, the hyperparameters of topic distribution in the document $(\alpha)$, and word distribution in the topic $(\beta)$. The topic assigned for the word in the document is defined as $z_{d, n}$, and $w_{d, n}$ is the words observed in the document.

LDA employs two different processes, generative and inferential. This study used the inference process, in which the parameters of word distribution in the topic $\left(\varphi_{k}\right)$ and the proportion of topics for each document $\left(\theta_{d, n}\right)$ are identified, as well as word distribution among the number of topics based on the available document (Putri \& Kusumaningrum, 2017). The parameters for this study were the number of topics, number of iterations, and the alpha $(\alpha)$ hyperparameters. The number of topics varied between five and nine, while the number of iterations were 2000, 3000, 4000 and 5000. The alpha $(\alpha)$ hyperparameters were set to 0.1 and 0.01 .

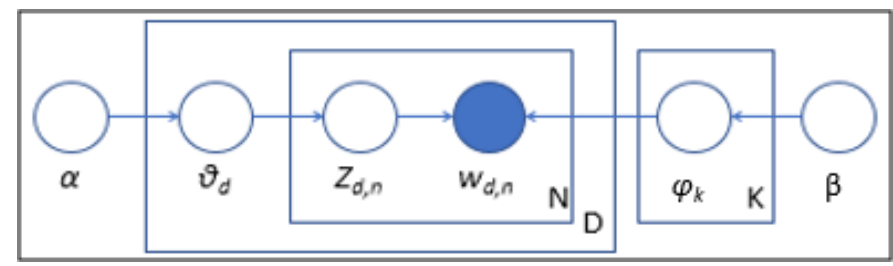

Figure 1 Graphical model of LDA

For the evaluation process, perplexity value was employed. This value is used to define how well a probability model predicts a sample. A probability model can predict a sample well if it has a low perplexity value. The topics were extracted from the model with parameter combination, resulting in the least perplex value. The equation for calculating perplexity is as follows (Gruen \& Hornik, 2011):

$$
\operatorname{Perplexity}(w)=\exp \left\{\frac{\log (p(w))}{\sum_{d=1}^{D} \sum_{j=1}^{V} n^{(j d)}}\right\}
$$

where $n^{(j d)}$ is the frequency of occurrence of $j^{\text {th }}$ word in $d^{\text {th }}$ document.

\subsection{Sentiment Analysis and Classification}

The study used the Support Vector Machine (SVM), which is a supervised classification algorithm. SVM finds the optimum hyperplane separating the dataset by maximizing the closest distance between the classes, as shown in Figure 2 (Han et al., 2012). Hyperplanes with larger margins have a higher accuracy due to their ability to separate the data by finding the farthest separators between groups.

SVM was employed to classify customer tweets based on six predefined categories with their respective polarities. There were more than two classes in the classification, so the approach used for SVM should be multi-class. There are two commonly used methods for multi-class classification: one-versus-all and one-versus-one. This study used the one-versus-one method, which evaluates all the possible binary classifications and gives a final class label for test data based on a majority vote. Besides, one-versus-one provides better performance than one-versusall because it produces more symmetrical results (Hsu \& Lin, 2002; Wang \& Xue, 2014). 
The classification model was developed using a machine learning scheme. The data were divided into training and testing data; however, the number of negative tweets in this study was more than positive ones. Therefore, random oversampling was conducted to make the training data more balanced. The percentages of resampling for the tweet data of the three ride-hailing service providers were $47 \%, 44 \%$, and $32 \%$, respectively, due to the varying numbers of data. Once created, the classification model was used for processing new data that had not been given class labels.

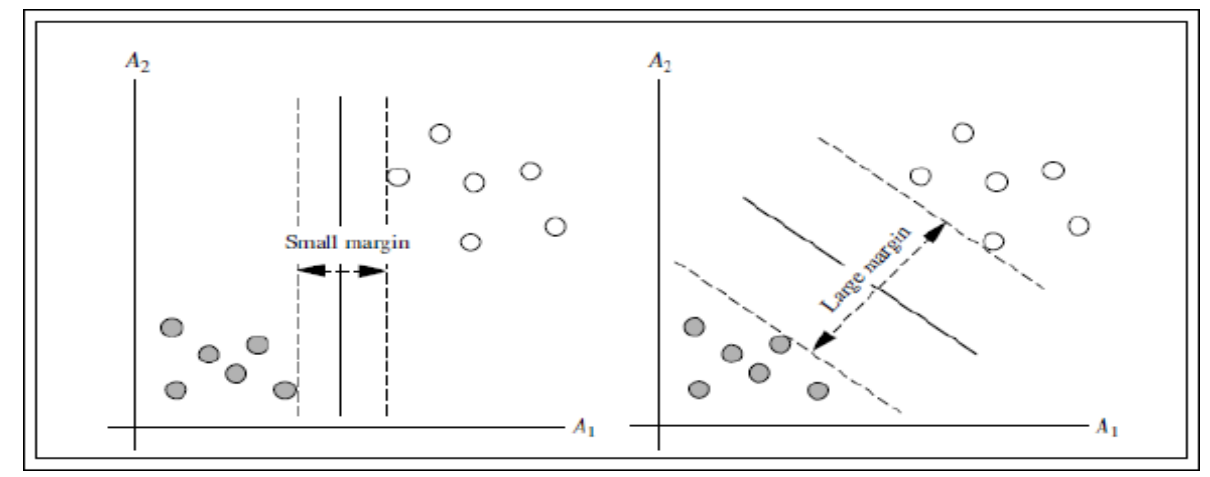

Figure 2 Hyperplane in SVM

\subsection{Ride-Hailing Service Assessment}

Assessment of the services provided by the ride-hailing service providers was made based on the sentiment analysis results. After the number of positive and negative tweets on each topic for each service provider had been obtained, the Net Reputation Score (NRS) method was used. This method generates scores based on the percentage of positive and negative comments given by customers (Social Meteor, 2017).

The scores ranged from $100 \%$ to $-100 \%$, depending on the positive and negative comments. They can be used to establish the quality of service according to customers' points of view in the social media, so that consideration of the strategies to maintain or improve quality can be defined.

\section{RESULTS AND ANALYSIS}

\subsection{Topic Modeling Results}

The lowest perplexity value of all the parameter combinations for the first service provider was 227.44. This was generated from the combination of six topics, 4000 iterations, and an $\alpha$ hyperparameter of 0.01. The topics for the first service provider can be seen in Table 4 .

For the second service provider, the lowest perplexity value of 205.86 was derived from a combination of six topics, 3000 iterations, and an $\alpha$ hyperparameter of 0.1 . Table 5 shows the topics for this provider.

The lowest perplexity value obtained from the topic modeling of the third service provider was 163.87. In this case, the combination consisted of six topics, 3000 iterations, and a 0.1 hyperparameter. The topics for the third service provider can be seen in Table 6 .

The number of topics that produced the lowest perplexity value was six. This number was also chosen because if there had been more topics, similar or related topics could have appeared more than once, so the number of members of each topic would have decreased. On the other hand, smaller $\alpha$ values indicate that the topics distributed within the documents were fewer, thus requiring the support of other parameters to achieve a more stable distribution, while larger $\alpha$ values indicate that the distribution of topics in the document is more even and stable. 
Iteration shows the number of perplexity calculations until the optimal value is reached. The greater the number of words in the document, the more iterations it will take to appropriately generate the word distribution model of each topic in the document. This is in line with the results of the topic modeling in this study, because the number of tweets in relation to the first service provider was greater than those for the second and third, so it went through more iterations.

Table 4 Topics for the first service provider

\begin{tabular}{cl}
\hline No. & \multicolumn{1}{c}{ Topic } \\
\hline 1 & Transportation Service \\
2 & Service Fares \\
3 & Electronic Money, Rewards and Credit System \\
4 & Instant Courier Service \\
5 & Food Delivery Service \\
6 & App Security and Stability \\
\hline
\end{tabular}

Table 5 Topics for the second service provider

\begin{tabular}{cl}
\hline No. & \multicolumn{1}{c}{ Topic } \\
\hline 1 & Electronic Money System \\
2 & Transportation Service \\
3 & Service Fares and Promotions \\
4 & App Stability \\
5 & Instant Courier Service \\
6 & Food Delivery Service \\
\hline
\end{tabular}

Table 6 Topics for the third service provider

\begin{tabular}{cl}
\hline No. & \multicolumn{1}{c}{ Topic } \\
\hline 1 & Non-Cash Payment \\
2 & Transportation Service \\
3 & Promotions and Discounts \\
4 & Services Ordering in App \\
5 & Order Cancellation Policy \\
6 & Service Fares \\
\hline
\end{tabular}

\subsection{Sentiment Analysis Results}

The tweets for each service provider were classified based on the topics generated at the topic modeling stage. Each topic was divided based on its positive and negative sentiments. The classification model was generated with the one-versus-all SVM algorithm.

The classification results can be obtained by calculating the level of accuracy, precision, recall and F1 after processing the test data (Rokach \& Maimon, 2015). The accuracy value represents the proportion of the number of documents classified correctly from the entire document. Precision and recall are used to evaluate the model results of each class, and F1 is a single measure of precision and recall.

The percentages of training and testing data specified in the model development were $70 \%$ and $30 \%$ respectively. The three classification models resulted in fairly good accuracy of $86 \%$ for the first service provider, $91 \%$ for the second, and $87 \%$ for the third. The precision, recall and F1 values for most classes exceeded $80 \%$.

In the classification model for the first service provider, the topic of transportation service attracting positive sentiment had a precision value of only $63 \%$ and an F1 value of $68 \%$. This may be because the process of labeling tweets into topics and sentiments was done manually, or 
because the comparison of the number of positive and negative sentiments was not balanced in the topic.

\subsection{Service Assessment Results}

The previously generated classification models were used for the classification of new data so that the number of tweets for each topic and sentiment could be known. The numbers were used to provide an assessment of the three service providers as a whole, based on the topic by the NRS method. The assessment of each service provider can be seen in Tables 7, 8 and 9 .

Assessment of the three ride-hailing service providers shows that all the topics have negative NRS. This happened because there were more tweets with negative sentiments than the positive ones. Transportation service from the first service provider achieved the highest score, while the security and stability of the app achieved the lowest. For the second service provider, service fares and promotions obtained the highest score, with the instant courier service receiving the lowest. The topic that achieved the highest score for the third service provider was promotions and discounts, while those which obtained the lowest scores were non-cash payment, service ordering in the app, and order cancellation policy.

Table 7 NRS scores for the first service provider

\begin{tabular}{clccc}
\hline \multirow{2}{*}{ No. } & \multicolumn{1}{c}{ Topic } & \multicolumn{2}{c}{ Number of Tweets } & Net Reputation \\
\cline { 3 - 4 } & & Positive & Negative & Score \\
\hline 1 & Transportation Service & 24 & 152 & $-72.72 \%$ \\
2 & Service Fares & 6 & 149 & $-92.26 \%$ \\
3 & Electronic Money, Rewards and & 13 & 305 & $-91.82 \%$ \\
& Credit System & & & \\
4 & Instant Courier Service & 4 & 127 & $-93.89 \%$ \\
5 & Food Delivery Service & 10 & 73 & $-75.90 \%$ \\
6 & App Security and Stability & 11 & 409 & $-94.76 \%$ \\
\hline & $\quad$ Total & 68 & 1215 & $-89.39 \%$ \\
\hline
\end{tabular}

Table 8 NRS scores for the second service provider

\begin{tabular}{clccc}
\hline \multirow{2}{*}{ No. } & \multicolumn{1}{c}{ Topic } & \multicolumn{2}{c}{ Number of Tweets } & \multirow{2}{*}{$\begin{array}{c}\text { Net Reputation } \\
\text { Score }\end{array}$} \\
\cline { 3 - 4 } & & Positive & Negative & $-99.06 \%$ \\
\hline 1 & Electronic Money System & 3 & 634 & $-92.14 \%$ \\
2 & Transportation Service & 25 & 611 & $-54.95 \%$ \\
3 & Service Fares and Promotions & 25 & 86 & $-98.58 \%$ \\
4 & App Stability & 1 & 140 & $-100 \%$ \\
5 & Instant Courier Service & 0 & 71 & $-27.78 \%$ \\
6 & Food Delivery Service & 13 & 23 & $-91.79 \%$ \\
\hline & Total & 67 & 1565 & \\
\hline
\end{tabular}

Table 9 NRS scores for the third service provider

\begin{tabular}{clccc}
\hline \multirow{2}{*}{ No. } & \multicolumn{1}{c}{ Topic } & \multicolumn{2}{c}{ Number of Tweets } & \multirow{2}{*}{$\begin{array}{c}\text { Net Reputation } \\
\text { Score }\end{array}$} \\
\cline { 3 - 4 } & Positive & Negative & $-100 \%$ \\
\hline 1 & Non-Cash Payment & 0 & 17 & $-96.59 \%$ \\
2 & Transportation Service & 7 & 404 & $-50 \%$ \\
3 & Promotions and Discounts & 3 & 9 & $-100 \%$ \\
4 & Service Ordering in the App & 0 & 125 & $-100 \%$ \\
5 & Order Cancellation Policy & 0 & 124 & $-80.95 \%$ \\
6 & Service Fares & 2 & 19 & \\
\hline
\end{tabular}


In all the topics, there was similarity between all the ride-hailing service providers in terms of transportation services, service fares and app. In transportation services and app, the first service provider achieved the highest NRS, followed by the second and third providers. Customers considered the transportation service of the first service provider to be more convenient and to have more professional drivers, while noting that the app had a new interface and chat features. Meanwhile, the second service provider had the highest NRS compared to the other two in terms of service fares because these were more stable and affordable. The second service provider also conducted regular promotions.

In addition to these three topics, the first and second service providers also shared the same topics of electronic money system, food delivery services, and instant courier services. The electronic money system and instant courier services of the first service provider had a better reputation amongst the customers. However, the second service provider was considered better at food delivery services.

From the total NRS value, the first ride-hailing service provider achieved the highest score, followed by the second and third. This means that the first provider has a better reputation than its two competitors because customers made positive comments on all the topics generated. However, there were still a large number of tweets with negative sentiments for the three service providers. Therefore, since the third provider has been acquired, the first and second providers need to evaluate and improve the services they provide.

\section{CONCLUSION}

Ride-hailing services have many positive impacts on urban life. Therefore, it is not surprising that they are increasingly being used, and discussed on social media such as Twitter. The opinions and complaints of users on Twitter can be an input for service providers to evaluate and measure the quality of service provided according to the customers' points of view. To process text data on Twitter that is in large amounts, the text mining approach in the form of sentiment analysis can be used in the process of analyzing tweets from customers. This study combined the aspectbased sentiment analysis approach to identify topics in the customer opinions and their sentiments, with assessment of the ride-hailing service providers in general, and more specifically based on the topics and sentiments produced.

The topic modeling stage generated six topics regarding the services, apps and fares of all three ride-hailing service providers. Once the topics had been decided, positive and negative data classification by topic and sentiment was conducted. The classification model yielded an accuracy of $86 \%$ for the first service provider, $91 \%$ for the second, and $87 \%$ for the third. The model was used for the classification of new data to obtain the number of tweets with positive and negative sentiments for each topic used for the assessment of the three providers. The resulting scores were entirely negative because of the number of tweets that had more negative sentiments. However, based on the scores, the first service provider had a better reputation because there were customers who made positive comments on all the topics.

While the results could benefit the ride-hailing service providers, more time could have been taken in the text pre-processing phase of the approach employed. This is because of the immense variety of acronyms, spelling and even local language included in the text, while the availability of pre-processing software in Indonesian is still limited.

Development of this research could be made by adding opinions and complaints from the drivers, so that their needs and aspirations can also be fulfilled. In terms of the social media used, further 
research could add data from the comments columns in other social media such as Facebook or Instagram, or from reviews on Google Play Store or the App Store. Research could also be developed by comparing user opinions on services of the second service provider before and after acquiring the third provider. In terms of the algorithm used, further research could be made using other topic modeling techniques such as Latent Semantic Indexing (LSI) or Probabilistic Latent Semantic Analysis (PLSA), and other classification algorithms such as Decision Tree, Naïve Bayes or neural network. Finally, further research could be conducted to compare the online ridehailing service with public transportation, so that the advantages and disadvantages of each can be defined.

\section{REFERENCES}

Blei, D.M., 2012. Probabilistic Topic Model. Communications of the ACM, Volume 55(4), pp. $77-84$

Chakraborty, G., Pagolu, M., Garla, S., 2013. Text Mining and Analysis: Practical Methods, Examples, and Case Studies using SAS. Cary, North Carolina: SAS Publishing

Colace, F., Casaburi, L., De Santo, M., Greco, L., 2015. Sentiment Detection in Social Networks and in Collaborative Learning Environments. Computers in Human Behavior, Volume 51(B), pp. 1061-1067

Duan, J., Ai, Y., Li, X., 2015. LDA Topic Model for Microblog Recommendation. In: International Conference on Asian Language Processing, Suzhou

Gruen, B., Hornik, K., 2011. Topic Models: An R Package for Fitting Topic Models. Journal of Statistical Software, Volume 40(13), pp. 1-30

Han, J., Kamber, M., Pei, J., 2012. Data Mining: Concepts and Techniques. $3^{\text {rd }}$ Edition. San Fransisco: Morgan Kaufmann Publishers

He, W., Zha, S., Li, L., 2013. Social Media Competitive Analysis and Text Mining: A Case Study in the Pizza Industry. International Journal of Information Management, Volume 33(3), pp. 464-472

Hsu, C.W., Lin, C.J., 2002. A Comparison of Methods for Multiclass Support Vector Machines. In: IEEE Transactions on Neural Networks, Taiwan, pp. 415-425

Lim, K.W., Buntine, W., 2012. Twitter Opinion Topic Model: Extracting Product Opinions from Tweets by Leveraging Hashtags and Sentiment Lexicon. In: The $21^{\text {st }}$ ACM International Conference on Information and Knowledge Management, Maui

Liu, Y., Bi, J.W., Fan, Z.P., 2017. Ranking Products Through Online Reviews: A Method based on Sentiment Analysis Technique and Intuitionistic Fuzzy Set Theory. Information Fusion, Volume 36, pp. 149-161

Marrese-Taylor, E., Velásquez, J.D., Bravo-Marquez, F., 2014. A Novel Deterministic Approach for Aspect-based Opinion Mining in Tourism Products Reviews. Expert Systems with Applications, Volume 41, pp. 7764-7775

Miner, G., Elder IV, J., Fast, A., Hill, T., Nisbet, R., Delen, D., 2012. Practical Text Mining and Statistical Analysis for Non-structured Text Data Applications. Oxford: Elsevier.

Philander, K., Zhong, Y., 2016. Twitter Sentiment Analysis: Capturing Sentiment from Integrated Resort Tweets. International Journal of Hospitality Management, Volume 55, pp. $16-24$

Prameswari, P., Surjandari, I., Zulkarnain, Laoh, E., 2017. Mining Online Reviews in Indonesia's Priority Tourist Destinations using Sentiment Analysis and Text Summarization Approach. In: IEEE $8^{\text {th }}$ International Conference on Awareness Science and Technology, Kaohsiung

Putri, I.R., Kusumaningrum, R., 2017. Latent Dirichlet Allocation (LDA) for Sentiment Analysis Toward Tourism Review in Indonesia. Journal of Physics: Conference Series, Volume 801(1), pp. 1-6 
Rokach, L., Maimon, O., 2015. Data Mining with Decision Trees: Theory and Applications. $2^{\text {nd }}$ Edition. Singapore: World Scientific Publishing

Social Meteor, 2017. Net Reputation Score: Dropping Neutral Mentions Means More. Available Online at: http://www.socialmeteor.com/2017/03/16/net-reputation-score-dropping-neutralmeans/, Accessed on March 16 $6^{\text {th }}, 2017$

Surjandari, I., Megawati, C., Dhini, A., Hardaya, I.B.N.S., 2016. Application of Text Mining for Classification of Textual Reports: A Study of Indonesia's National Complaint Handling System. In: Sixth International Conference on Industrial Engineering and Operations Management, Kuala Lumpur

Surjandari, I., Naffisah, M.S., Prawiradinata, M.I., 2015. Text Mining of Twitter Data for Public Sentiment Analysis of Staple Foods Price Changes. Journal of Industrial and Intelligent Information, Volume 3(3), pp. 253-257

Tang, J., Liu, H., 2014. Feature Selection for Social Media Data. ACM Transactions on Knowledge Discovery from Data (TKDD), Volume 8(4), pp. 1-27

Tong, Z., Zhang, H., 2016. A Text Mining Research Based on LDA Topic Modelling. In: The Sixth International Conference on Computer Science, Engineering and Information Technology, Vienna

Vidya, N.A., Fanany, M.I., Budi, I., 2015. Twitter Sentiment to Analyze Net Brand Reputation of Mobile Phone Providers. Procedia Computer Science, Volume 72, pp. 519-526

Wang, Z., Xue, X., 2014. Multi-class Support Vector Machines. Support Vector Machines Applications. Basel: Springer International Publishing, pp. 23-49 